\title{
Phenotypic and genotypic correlation of carbapenememase-producing Enterobacteriaceae and problems experienced in routine screening
}

\author{
A Singh-Moodley, ${ }^{1,2}$ BSc, BMedSc Hons, MMedSc, PhD; O Perovic, ${ }^{1,2}$ MD, DTM\&H, FC Path (SA) (Microbiol), MMed (Microbiol) \\ ${ }^{1}$ Centre for Healthcare-associated infections, Antimicrobial Resistance and Mycoses, National Institute for Communicable Diseases, National \\ Health Laboratory Service, Johannesburg, South Africa \\ ${ }^{2}$ Department of Clinical Microbiology and Infectious Diseases, School of Pathology, Faculty of Health Sciences, University of the Witwatersrand, \\ Johannesburg, South Africa
}

Corresponding author: A Singh-Moodley (ashikas@nicd.ac.za)

\begin{abstract}
Background. The emergence and transmission of carbapenem-resistant Enterobacteriaceae (CRE) is a concern in both the clinical and public health arenas. Reliable and accurate detection of these organisms is required for patient management and infection prevention and control purposes. In the routine laboratory, phenotypic methods are utilised for identification of CRE.

Objectives. To investigate the phenotypic profiles of suspected carbapenemase-producing Enterobacteriaceae (CPE) isolates generated by the automated MicroScan Walkaway system making use of the Clinical and Laboratory Standards Institute (CLSI) guidelines, and correlate these with carbapenemase production by molecular methods.

Methods. Antimicrobial susceptibility testing was performed using the MicroScan Walkaway system, and the presence of six carbapenemase genes $\left(b l a_{\mathrm{NDM}}, b l a_{\mathrm{VIM}}, b l a_{\mathrm{IMP}}, b l a_{\mathrm{OXA}-48}\right.$ and variants, $b l a_{\mathrm{GES}}$ and $\left.b l a_{\mathrm{KPC}}\right)$ was screened for using a multiplex real-time polymerase chain reaction. Results. A total of 2678 isolates were evaluated. Klebsiella pneumoniae accounted for $62.9 \%$ of the isolates $(n=1685)$, followed by Enterobacter cloacae $(n=361,13.5 \%)$. Carbapenemases accounted for $75.2 \%$ of isolates; $b l a_{\mathrm{OXA}-48}$ and its variants predominated $(n=978$, $36.5 \%)$, followed by $b l a_{\mathrm{NDM}}(n=904,33.8 \%), b l a_{\mathrm{VIM}}(n=108,4.0 \%), b l a_{\mathrm{IMP}}(n=35,1.3 \%), b l a_{\mathrm{GES}}(n=24,0.9 \%)$ and $b l a_{\mathrm{KPC}}(n=18,0.7 \%)$.

Conclusions. A considerable number of isolates expressing a carbapenemase or carbapenemases (the majority of which were $b l a_{\mathrm{OXA}-48}$ producing) were susceptible to third-and fourth-generation cephalosporins and carbapenems, demonstrating that confirmed carbapenemase-producing isolates are not presenting as possible carriers of carbapenemases using routine diagnostic methods. Similar results were obtained when CLSI and European Committee on Antimicrobial Susceptibility Testing (EUCAST) clinical breakpoints were applied and are suitable for the purpose of patient management. However, since genotyping assays are costly, it is suggested that routine laboratories first perform comprehensive phenotypic screening for CPE.
\end{abstract}

S Afr Med J 2018;108(6):495-501. DOI:10.7196/SAMJ.2018.v108i6.12878

Multidrug-resistant organisms are a global clinical and public health concern. ${ }^{[1,2]}$ There is an increase in the detection of Enterobacteriaceae strains with resistance observed against betalactams, fluoroquinolones, aminoglycosides and polymyxins. ${ }^{[3]}$ Resistance mechanisms to the beta-lactams are multifaceted and include outer-membrane permeability changes, upregulation of efflux pumps, the production of an inducible ampicillin class $\mathrm{C}(\mathrm{AmpC})$ beta-lactamase, and enzymes that hydrolyse the antimicrobial agent. Beta-lactamases are enzymes that include the cephalosporinases, such as the extended-spectrum beta-lactamases (ESBLs) and the carbapenemases, namely the metallo-beta-lactamases (MBLs), which consist of New Delhi MBLs (NDMs), IMPs (imipenemases) and VIMs (Verona integron-encoded MBLs), the Klebsiella pneumoniae carbapenemases (KPCs) and the oxacillinases such as OXA-48. ${ }^{[3]}$

It is important to note the difference between carbapenemresistant Enterobacteriaceae (CRE) and carbapenemase-producing Enterobacteriaceae (CPE). CRE are resistant to carbapenems due to carbapenemase production or via combinations of AmpC, ESBLs and impermeability. These multiple resistance mechanisms may play a role in acquiring or expressing carbapenem resistance. CPE include only those Enterobacteriaceae with a confirmed carbapenemaseproducing gene. ${ }^{[4]}$ These genes code for the enzymes, carbapenemases, which are beta-lactamases that hydrolyse almost all beta-lactams including the carbapenems. These enzymes have been classified into Ambler classes A, B and D based on their amino acid sequences. ${ }^{[3,5]}$ Class A carbapenemases include KPC and Guiana extended-spectrum beta-lactamase (GES), class B carbapenamases include MBLs, such as VIMs, NDMs and IMPs, and class D consists of OXA-48 and its derivatives. ${ }^{[6,7]}$ Carbapenemases were initially chromosomally mediated (mutation within the bacterial chromosome) resulting in resistance in a few specific species, but the majority are now plasmid mediated ${ }^{[8,9]}$ resulting in horizontal transmission among various bacterial species and genera, i.e. transfer from one organism to another. As plasmids are mobile genetic elements (DNA that move around within the genome), their ability to acquire, harbour and disseminate multiple resistance genes contributes to the successful and aggressive transmission of resistant organisms. ${ }^{[10]}$

It is important to prevent the spread of these organisms. However, there are some obstacles, as clinical laboratories do not routinely test for CRE resistance mechanisms and are not able to differentiate between CRE and CPE ${ }^{[11]}$ Clinical routine laboratories make use of phenotypic methods that involve identification of the organism and antimicrobial susceptibility testing (AST). This provides the clinician with information on the organism causing the infection and the 
antibiotics to which it is susceptible or resistant, but not the actual mechanism causing the resistance. Molecular methods (also known as genotypic methods) provide this additional information, as they identify the specific resistance genes. Another obstacle in detecting these organisms has been emphasised by studies showing that CPE can be classified as phenotypically susceptible, making their identification all the more challenging, ${ }^{[12,13]}$ as carbapenem minimal inhibitory concentrations (MICs) for CPE may be below the clinical breakpoints, which are discriminatory antimicrobial concentrations used in the interpretation of results for susceptibility testing to define isolates as susceptible, intermediate or resistant. ${ }^{[14]}$ This means that although a carbapenemase-producing gene may be present, phenotypic AST results display no resistance because routinely used automated AST systems cannot identify very low MICs. It is suggested that because the European Committee on Antimicrobial Susceptibility Testing (EUCAST) breakpoints are higher than those recommended by the Clinical and Laboratory Standards Institute (CLSI), some CPE that are susceptible are taken into consideration. ${ }^{[15-18]}$ Currently, automated systems are not able to detect all types of carbapenemase producers reliably, ${ }^{[17]}$ particularly those exhibiting low MICs, as lower MIC dilutions are not included in the methodology of these systems. This highlights the need to understand the prevalence of these organisms in our current setting and to establish a guide that would be useful for identification of the organisms for clinical management and public health and infection prevention and control purposes.

\section{Objectives}

We evaluated 2678 Enterobacteriaceae isolates and correlated their phenotypic and genotypic profiles, with the objective of making recommendations for the identification and detection of CPE for both patient management and epidemiological and infection prevention and control purposes.

\section{Methods}

Organism identification and AST were performed using automated systems (VITEK II (bioMèrieux, France) and/or the Microflex MALDI-ToF (Bruker Daltonik GmbH, Germany) and the MicroScan Walkaway system (Siemens, USA)). The interpretation of susceptibility was done according to the CLSI guidelines ${ }^{[19]}$ and the EUCAST guidelines. ${ }^{[20]}$ DNA was extracted using a crude boiling method at $95^{\circ} \mathrm{C}$ for 25 minutes for cell lysis. The supernatant was harvested and screened for $b l a_{\mathrm{NDM}}, b l a_{\mathrm{KPC}}, b l a_{\mathrm{OXA}-48}$ and its variants (OXA-162, 163, 244, 245, 247, 181, 204, 232), bla $a_{\text {GES }}$ (GES-1-9, 11) $b l a_{\mathrm{IMP}}(\mathrm{IMP}-9,16,18,22,25)$ and $b \operatorname{la}_{\mathrm{VIM}}$ (VIM-1-36) using a multiplex real-time polymerase chain reaction (LightCycler 480 II, Roche Diagnostics, USA), the LightCycler 480 Probes Master kit (Roche Diagnostics, USA) and the individual LightMix Modular kits (Roche Diagnostics, USA).

\section{Ethical approval}

Permission to conduct this study was obtained from the Human Research Ethics Committee (Medical) R14/49, University of the Witwatersrand, Johannesburg, South Africa (SA) (ref. no. M10464). General ethics approval was obtained for routine diagnostic testing, and patient consent was not required. No personal individual information was used.

\section{Results}

A total of 2678 isolates were evaluated. Isolates were received as part of a referral diagnostic programme and processed in real time. A breakdown of the organisms isolated and specimen types is seen in Table 1. A total of 2014 isolates $(75.2 \%)$ harboured one or a combination of carbapenemases. $B l a_{\mathrm{OXA}-48}$ and its variants predominated $(n=978,36.5 \%)$ followed by $b l a_{\mathrm{NDM}}(n=904,33.8 \%)$, $b l a_{\mathrm{VIM}}(n=108,4.0 \%), b l a_{\mathrm{IMP}}(n=35,1.3 \%), b l a_{\mathrm{GES}}(n=24,0.9 \%)$ and $b l a_{\mathrm{KPC}}(n=18,0.7 \%)$. The majority of the carbapenemase-producing isolates were Klebsiella spp. ( $n=1459,54.5 \%)$, driven predominantly by K. pneumoniae ( $n=1413,52.8 \%$ ), followed by Enterobacter spp. ( $n=212,7.9 \%)$ with E. cloacae $(n=170,6.3 \%)$ being the predominant species (Fig. 1). Tables 2 and 3 show the MIC breakpoint values for the cephalosporins and carbapenems. As observed in these tables, a considerable number of isolates expressing a carbapenemase or carbapenemases were susceptible to third- and fourth-generation cephalosporins and the carbapenem group of antimicrobial agents when the CLSI ${ }^{[19]}$ and EUCAST ${ }^{[20]}$ guideline clinical breakpoints were applied, thus indicating the number of isolates that would have been missed using the respective guidelines. There was no difference in the percentage of susceptible isolates expressing a carbapenemase or carbapenemases for cefotaxime, ertapenem, meropenem and doripenem when analysed using both guideline clinical breakpoints. Differences were seen with ceftazidime, which showed a $2 \%$ increase in the number of susceptible isolates expressing a carbapenemase

Table 1. Distribution of organism and specimen type $(N=2678)$

\begin{tabular}{|c|c|}
\hline & $n(\%)$ \\
\hline \multicolumn{2}{|l|}{ Organism type } \\
\hline Escherichia coli & $178(6.6)$ \\
\hline Serratia marcescens & $146(5.5)$ \\
\hline Morganella morganii & $28(1.0)$ \\
\hline Enterobacter aerogenes & $27(1.0)$ \\
\hline E. asburiae & $12(0.4)$ \\
\hline E. cancerogenus & $1(0.0)$ \\
\hline E. cloacae & $416(15.5)$ \\
\hline E. gergoviae & $1(0.0)$ \\
\hline E. kobei & $8(0.3)$ \\
\hline Enterobacter spp. & $3(0.1)$ \\
\hline Klebsiella pneumoniae & $1685(62.9)$ \\
\hline K. oxytoca & $30(1.1)$ \\
\hline Klebsiella spp. & $18(0.7)$ \\
\hline Providencia spp. & 46() 1.7 \\
\hline Raoultella spp. & $3(0.1)$ \\
\hline Proteus spp. & 11() 0.4 \\
\hline Citrobacter freundii & $58(2.2)$ \\
\hline Citrobacter spp. & $7(0.3)$ \\
\hline \multicolumn{2}{|l|}{ Specimen type } \\
\hline Unknown & $525(19.6)$ \\
\hline Blood & $398(14.9)$ \\
\hline Body fluids & $142(5.3)$ \\
\hline Bone marrow biopsy & $1(0.0)$ \\
\hline Catheter tip vascular & $128(4.8)$ \\
\hline Cerebrospinal fluid & $10(0.4)$ \\
\hline Drain & $3(0.1)$ \\
\hline Lower respiratory tract aspirate & $82(3.1)$ \\
\hline Sputum & $91(3.4)$ \\
\hline Sterile site aspirate & $14(0.5)$ \\
\hline Stool & $18(0.7)$ \\
\hline Swabs & $539(20.1)$ \\
\hline Tissue & $55(2.1)$ \\
\hline Upper respiratory tract swab & $16(0.6)$ \\
\hline Urinary catheter & $34(1.3)$ \\
\hline Urine & $622(23.2)$ \\
\hline
\end{tabular}




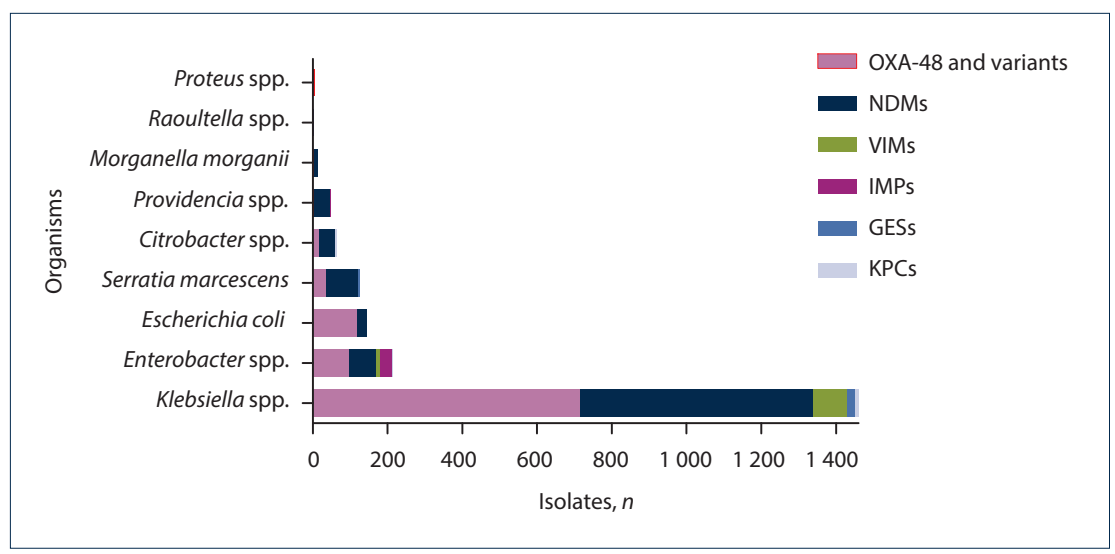

Fig. 1. Distribution of organism and carbapenemase types.

Proteus spp.: OXA-48, $\mathrm{n}=4$; NDM, $\mathrm{n}=1$

Raoultella spp.: NDMs, $\mathrm{n}=2 ; V I M, \mathrm{n}=1$

Morganella morganii: $O X A-48, \mathrm{n}=1 ; N D M s, \mathrm{n}=11$

Providencia spp.: OXA-48, $\mathrm{n}=2 ; N D M s, \mathrm{n}=42 ; V I M, \mathrm{n}=1 ; I M P s, \mathrm{n}=2$

Citrobacter spp.: OXA-48, $\mathrm{n}=14 ;$ NDMs, $\mathrm{n}=43$; GES, $\mathrm{n}=1 ; K P C s, \mathrm{n}=4$

Serratia marcescens: $O X A-48, \mathrm{n}=34 ; N D M s, \mathrm{n}=85 ;$ VIM, $\mathrm{n}=1 ;$ GESs, $\mathrm{n}=4$

Escherichia coli: $O X A-48, \mathrm{n}=117 ; N D M s, \mathrm{n}=25 ;$ VIM, $\mathrm{n}=1$

Enterobacter spp.: OXA-48, $\mathrm{n}=94$; NDMs, $\mathrm{n}=72$; VIMs, $\mathrm{n}=12 ;$ GESs, $\mathrm{n}=31 ;$ KPCs, $\mathrm{n}=3$

Klebsiella spp.: OXA-48, $\mathrm{n}=712$; NDMs, $\mathrm{n}=623$; VIMs, $\mathrm{n}=92 ;$ IMPs, $\mathrm{n}=2 ;$ GESs, $\mathrm{n}=19 ;$ KPCs, $\mathrm{n}=11$

$(M B L s=$ metallo-beta-lactamases; NDMs = New Delhi MBLs; VIMs = Verona integron-encoded MBLs; IMPs = imipenemases; GESs = Guiana extended-spectrum beta-lactamases; KPCs = Klebsiella pneumoniae carbapenemases.)

or carbapenemases using EUCAST clinical breakpoints, cefepime, which showed a $1 \%$ decrease, and imipenem, which showed a $4 \%$ decrease. From these data it is further observed that although all six genes were present in the clinically susceptible isolates, the majority of these isolates were $b l a_{\text {OXA-48 }}$ producing.

\section{Discussion}

This study demonstrates the problems experienced in the detection of CPE. Overall $75.2 \%$ of the isolates harboured at least one or a combination of carbapenemaseproducing genes, although a substantial number of isolates were phenotypically susceptible. The most predominant carbapenemase type was $b \operatorname{la}_{\mathrm{OXA}-48}$ and its variants $(n=978,36.5 \%)$, followed by $b l a_{\mathrm{NDM}}$ $(n=904,33.8 \%), b l a_{\mathrm{VIM}}(n=108,4.0 \%), b l a_{\mathrm{IMP}}$ $(n=35,1.3 \%), b l a_{\mathrm{GES}}(n=24,0.9 \%)$ and $b l a_{\mathrm{KPC}}$ $(n=18,0.7 \%)$. Limitations of the genotypic method employed should be noted; falsenegative results may have occurred because genes were not detected owing to the presence of rare carbapenemase types and/ or genetic variants that were not included in the screening assay, namely IMI, SPM, SIM, etc., suggesting that variants and other carbapenemases may potentially have been missed and that the actual proportion of CPE in our study population could have been higher. This is concerning, as the rate of CPE is increasing as demonstrated by the number of SA studies reporting occurrences and outbreaks. ${ }^{[13,21-25]}$

The majority of the carbapenemaseproducing isolates were $K$. pneumoniae ( $n=1413,52.8 \%)$, followed by E. cloacae $(n=170,6.3 \%)$. Carbapenem resistance is particularly worrying in intrinsically colistin-resistant organisms such as Serratia marcescens, Providencia spp. and Morganella morganii, which harboured carbapenemases in $124(4.6 \%), 47(1.8 \%)$ and $12(0.5 \%)$ isolates, respectively, and accounted for $6.3 \%$ of all isolates. Treatment options for patients infected with these isolates are limited, with most conventional treatment options being exhausted.

A considerable number of isolates producing a phenotypically susceptible profile to the third- and fourth-generation cephalosporins and carbapenems expressed carbapenemases. The numbers varied per antimicrobial agent (Tables 2 and 3), but were relatively high. Difficulties in identifying metallo-beta-carbapenemaseproducing organisms primarily based on MIC results have been reported previously. ${ }^{[12]}$ The most striking carbapenemase type in our study was OXA-48 and its variants. This is not surprising, as this genotype has an atypical hydrolysis profile and clinical isolates harbouring this gene rarely show the resistance phenotype that is attributable to the expression of carbapenemases alone, often expressing other beta-lactamases such as the
ESBLs, resulting in an extensive resistance profile. ${ }^{[1]]}$ However, intermediate resistance and susceptibility to carbapenems have been observed in various carbapenemase typeproducing Enterobacteriaceae, particularly OXA-48/OXA181-producing isolates that do not co-produce an ESBL. Therefore, any Enterobacteriaceae isolate with a decreased carbapenem susceptibility may potentially be considered for screening. ${ }^{[17]}$ Furthermore, a study of OXA-48-producer-infected mice has shown that ertapenem and imipenem failed to cure the rodents, even though the MIC values were low. ${ }^{[2]}$ This is concerning, as OXA-48 was the predominant genotype in our sample population, indicating that isolates are not presenting as possible carriers of carbapenemases using routine diagnostic methods. A previous report in Enterobacteriaceae with reduced susceptibility to at least one carbapenem has shown that increased resistance to temocillin (MIC $>64 \mathrm{mg} / \mathrm{mL}$ ) and pipercillin-tazobactam may assist in identifying possible OXA- 48 producers. ${ }^{[27]}$ Other phenotypic tests include the modified Hodge test (MHT), which is able to detect carbapenemase producers and works well for the detection of KPCs and OXA-48 but is less sensitive to NDM producers. ${ }^{[17]}$ However, it is improved if zinc is included in the culture medium. ${ }^{[28]}$ The MHT often lacks specificity, particularly in the presence of high-level AmpC- or cefotaxime-type ESBL producers, and it is time-consuming, requiring $24-48$ hours. ${ }^{[17]}$ Inhibition of ethylenediamintetra-acetic acid (EDTA) may be used for identifying MBLs, e.g. the Etest MBL strip (bioMérieux, UK). Imipenem and imipenem/EDTA are efficient for this purpose, particularly if the MBL producers exhibit high-level resistance, but may be problematic for low-level MBL producers. Other inhibitors include boronic acid, specific for KPC detection in $K$. pneumoniae when performed with impenem or meropenem. These phenotypic tests may be efficient in detecting some carbapenemases, but false-positive results can be obtained, particularly in AmpC producers. ${ }^{[17]}$ It is therefore necessary to define an appropriate screening phenotypic method that is both reliable and accurate.

In a previous study, we showed the MicroScan Walkaway system coupled with the CLSI MIC breakpoint ranges as a method of carbapenemase production to have a high sensitivity (98\%) and a low specificity (13\%) using ertapenem. ${ }^{[29]}$ From our results in the present study, using CLSI MIC breakpoint ranges for ertapenem, 157 (57.3\%) (Table 2) carbapenemase-producing isolates were not detected as carbapenemase 
Table 2. MIC breakpoint values for the cephalosporins and carbapenems and CLSI guideline clinical breakpoint ${ }^{[19]}$ interpretation

\begin{tabular}{|c|c|c|c|c|c|c|c|c|}
\hline Antimicrobial agent & $\begin{array}{l}\text { MIC } \\
(\mathrm{mg} / \mathrm{mL})\end{array}$ & NDMs & $\begin{array}{l}\text { OXA-48 and } \\
\text { variants }\end{array}$ & VIMs & GESs & IMPs & KPCs & Total \\
\hline \multirow[t]{7}{*}{ Cefotaxime } & $\leq 1$ & 7 & 148 & 2 & - & - & - & 209 \\
\hline & 2 & - & 9 & - & - & - & - & 15 \\
\hline & 4 & - & 16 & - & 1 & 1 & - & 21 \\
\hline & 8 & 1 & 9 & 1 & - & - & - & 13 \\
\hline & 16 & 4 & 10 & - & - & - & - & 23 \\
\hline & 32 & 11 & 18 & 3 & 2 & 1 & - & 45 \\
\hline & $>32$ & 881 & 768 & 102 & 21 & 33 & 18 & 2352 \\
\hline \multirow[t]{6}{*}{ Ceftazidime } & $\leq 1$ & 7 & 162 & 2 & - & 1 & - & 227 \\
\hline & 2 & - & 11 & - & 1 & - & - & 17 \\
\hline & 4 & 2 & 7 & - & 1 & - & - & 17 \\
\hline & 8 & - & 19 & - & 1 & - & - & 29 \\
\hline & 16 & 3 & 88 & 2 & 2 & - & - & 117 \\
\hline & $>16$ & 892 & 691 & 104 & 19 & 34 & 18 & 2271 \\
\hline \multirow[t]{6}{*}{ Cefepime } & $\leq 1$ & 8 & 168 & 2 & 1 & 1 & - & 260 \\
\hline & 2 & 2 & 20 & 1 & - & - & - & 32 \\
\hline & 4 & 4 & 14 & - & - & 2 & - & 41 \\
\hline & 8 & 12 & 21 & 2 & 2 & 4 & - & 65 \\
\hline & 16 & 16 & 60 & 9 & 2 & 5 & - & 106 \\
\hline & $>16$ & 862 & 695 & 94 & 19 & 23 & 18 & 2174 \\
\hline \multirow[t]{6}{*}{ Ertapenem } & $\leq 0.5$ & 13 & 128 & 12 & 3 & 1 & - & 274 \\
\hline & 1 & 11 & 195 & 21 & & 3 & - & 324 \\
\hline & $>1$ & 727 & 608 & 57 & 13 & 6 & 10 & 1721 \\
\hline & 2 & - & 8 & 4 & & 7 & & 39 \\
\hline & 4 & 4 & 20 & 10 & 1 & 9 & 2 & 56 \\
\hline & $>4$ & 149 & 19 & 4 & 7 & 9 & 6 & 264 \\
\hline \multirow[t]{5}{*}{ Imipenem } & $\leq 1$ & 35 & 634 & 8 & 2 & 8 & 1 & 1050 \\
\hline & $\leq 2$ & 40 & 157 & 14 & 4 & 20 & 1 & 429 \\
\hline & 4 & 70 & 40 & 58 & 2 & 2 & 3 & 201 \\
\hline & 8 & 274 & 45 & 15 & 2 & 1 & 5 & 356 \\
\hline & $>8$ & 485 & 102 & 13 & 14 & 4 & 8 & 642 \\
\hline \multirow[t]{5}{*}{ Meropenem } & $\leq 1$ & 34 & 659 & 27 & 3 & 27 & 1 & 1150 \\
\hline & 2 & 18 & 77 & 15 & 1 & 1 & - & 169 \\
\hline & 4 & 49 & 60 & 21 & 1 & 2 & - & 216 \\
\hline & 8 & 82 & 38 & 27 & 1 & 2 & 4 & 205 \\
\hline & $>8$ & 721 & 144 & 18 & 18 & 3 & 13 & 938 \\
\hline \multirow[t]{5}{*}{ Doripenem } & $\begin{array}{l}\text { Not tested: } \\
\text { not included } \\
\text { in panel }\end{array}$ & 155 & 52 & 24 & 9 & 26 & 8 & 428 \\
\hline & $\leq 1$ & 29 & 669 & 16 & 2 & 8 & 1 & 1072 \\
\hline & 2 & 28 & 56 & 14 & - & - & - & 155 \\
\hline & 4 & 49 & 54 & 12 & 1 & 1 & 2 & 164 \\
\hline & $>4$ & 643 & 147 & 42 & 12 & & 7 & 859 \\
\hline
\end{tabular}

producers, contributing to false-negative results. The remaining antibiotics displayed a higher number of false negatives when both the CLSI ${ }^{[19]}$ and EUCAST ${ }^{[20]}$ MIC clinical breakpoint ranges were applied. Another study has shown ertapenem to be a good indicator for carbapenemase production, as the MICs are usually higher than those of the other carbapenems; however, they have also shown the specificity to be problematic. ${ }^{[17]}$ In 2015, Maurer et al. ${ }^{[16]}$ showed that CLSI clinical breakpoints for ertapenem and meropenem non-susceptibility displayed lower sensitivity than the EUCAST recommendation. Nevertheless, as recommended by EUCAST and published reports, CLSI and EUCAST guideline clinical breakpoints are sufficient for making treatment decisions, while carbapenemase detection and confirmation is recommended for epidemiological and infection prevention and control purposes. ${ }^{[17,20]}$ The latter can be achieved by making use of EUCAST epidemiological cut-off values, which are considerably lower than the clinical breakpoints and detect early resistance. ${ }^{[14]}$ The EUCAST guidelines for detection of resistance mechanisms and specific 


\begin{tabular}{|c|c|c|c|c|c|c|c|c|}
\hline Antimicrobial agent & $\begin{array}{l}\mathrm{MIC} \\
(\mathrm{mg} / \mathrm{mL})\end{array}$ & NDMs & $\begin{array}{l}\text { OXA- } 48 \text { and } \\
\text { variants }\end{array}$ & VIMs & GESs & IMPs & KPCs & Total \\
\hline \multirow[t]{7}{*}{ Cefotaxime } & $\leq 1$ & 7 & 148 & 2 & - & - & - & 209 \\
\hline & 2 & - & 9 & - & - & - & - & 15 \\
\hline & 4 & - & 16 & - & 1 & 1 & - & 21 \\
\hline & 8 & 1 & 9 & 1 & - & - & - & 13 \\
\hline & 16 & 4 & 10 & - & - & - & - & 23 \\
\hline & 32 & 11 & 18 & 3 & 2 & 1 & - & 45 \\
\hline & $>32$ & 881 & 768 & 102 & 21 & 33 & 18 & 2352 \\
\hline \multirow[t]{6}{*}{ Ceftazidime } & $\leq 1$ & 7 & 162 & 2 & - & 1 & - & 227 \\
\hline & 2 & - & 11 & - & 1 & - & - & 17 \\
\hline & 4 & 2 & 7 & - & 1 & - & - & 17 \\
\hline & 8 & - & 19 & - & 1 & - & - & 29 \\
\hline & 16 & 3 & 88 & 2 & 2 & - & - & 117 \\
\hline & $>16$ & 892 & 691 & 104 & 19 & 34 & 18 & 2271 \\
\hline \multirow[t]{6}{*}{ Cefepime } & $\leq 1$ & 8 & 168 & 2 & 1 & 1 & - & 260 \\
\hline & 2 & 2 & 20 & 1 & - & - & - & 32 \\
\hline & 4 & 4 & 14 & - & - & 2 & - & 41 \\
\hline & 8 & 12 & 21 & 2 & 2 & 4 & - & 65 \\
\hline & 16 & 16 & 60 & 9 & 2 & 5 & - & 106 \\
\hline & $>16$ & 862 & 695 & 94 & 19 & 23 & 18 & 2174 \\
\hline \multirow[t]{6}{*}{ Ertapenem } & $\leq 0.5$ & 13 & 128 & 12 & 3 & 1 & - & 274 \\
\hline & 1 & 11 & 195 & 21 & & 3 & - & 324 \\
\hline & $>1$ & 727 & 608 & 57 & 13 & 6 & 10 & 1721 \\
\hline & 2 & - & 8 & 4 & & 7 & & 39 \\
\hline & 4 & 4 & 20 & 10 & 1 & 9 & 2 & 56 \\
\hline & $>4$ & 149 & 19 & 4 & 7 & 9 & 6 & 264 \\
\hline \multirow[t]{5}{*}{ Imipenem } & $\leq 1$ & 35 & 634 & 8 & 2 & 8 & 1 & 1050 \\
\hline & $\leq 2$ & 40 & 157 & 14 & 4 & 20 & 1 & 429 \\
\hline & 4 & 70 & 40 & 58 & 2 & 2 & 3 & 201 \\
\hline & 8 & 274 & 45 & 15 & 2 & 1 & 5 & 356 \\
\hline & $>8$ & 485 & 102 & 13 & 14 & 4 & 8 & 642 \\
\hline \multirow[t]{5}{*}{ Meropenem } & $\leq 1$ & 34 & 659 & 27 & 3 & 27 & 1 & 1150 \\
\hline & 2 & 18 & 77 & 15 & 1 & 1 & - & 169 \\
\hline & 4 & 49 & 60 & 21 & 1 & 2 & - & 216 \\
\hline & 8 & 82 & 38 & 27 & 1 & 2 & 4 & 205 \\
\hline & $>8$ & 721 & 144 & 18 & 18 & 3 & 13 & 938 \\
\hline \multirow[t]{5}{*}{ Doripenem } & $\begin{array}{l}\text { Not tested: } \\
\text { not included } \\
\text { in panel }\end{array}$ & 155 & 52 & 24 & 9 & 26 & 8 & 428 \\
\hline & $\leq 1$ & 29 & 669 & 16 & 2 & 8 & 1 & 1072 \\
\hline & 2 & 28 & 56 & 14 & - & - & - & 155 \\
\hline & 4 & 49 & 54 & 12 & 1 & 1 & 2 & 164 \\
\hline & $>4$ & 643 & 147 & 42 & 12 & & 7 & 859 \\
\hline
\end{tabular}

resistances of clinical and/or epidemiological importance ${ }^{[14,30]}$ state that neither ertapenem nor imipenem are ideal agents, as ertapenem has a high sensitivity and low specificity and imipenem should not be used as a stand-alone screening agent because the separation between wild-type (i.e. susceptible) organisms and carbapenemase producers is poor. Meropenem is the recommended agent because it has the best compromise between sensitivity and specificity. This recommendation was further reiterated in a 2016 study by Fattouh et al. ${ }^{[15]}$ investigating the meropenem MIC distribution profile of a large number of Enterobacteriaceae isolates with reduced susceptibility to carbapenems. They showed that based on CLSI and EUCAST clinical breakpoint interpretive criteria, $14 \%$ and $20 \%$ of carbapenemase producers, respectively, displayed MICs within the susceptible range, while $86 \%$ and $98.4 \%$ of carbapenemase-producing isolates were identified using the CLSI and EUCAST screening recommendations, respectively. ${ }^{[15]}$ Screening recommendations are 
therefore recommended as the preferred guideline for epidemiological and infection prevention and control purposes, as they detect carbapenemase producers exhibiting low MICs; this means that lower MIC dilutions would need to be included for screening, and this poses a limitation in the current study. In order for us to be able to do this, dilutions lower than the lowest MIC dilution are required for analysis, and this was not achievable using the automated MicroScan Walkaway system. Although the number of missed carbapenemase-producing isolates would have been reduced and consequently the least number of CPE isolates would be misidentified as epidemiologically susceptible, the exact numbers could therefore not be quantified. Serial broth microdilution methods are the ideal and recommended method of choice. However, this method is tedious and requires expertise, as antibiotic concentrations and dilutions need to be extremely precise and accurate. An epidemiological cut-off value for meropenem of $0.125 \mathrm{mg} / \mathrm{mL}$ as endorsed by EUCAST ${ }^{[14]}$ maximises CPE detection and is therefore the recommended approach; however, this is only feasible in a region with low carbapenemase prevalence ${ }^{[15]}$ and is therefore not ideal in our settings owing to the high number of OXA-48- and NDMproducing Enterobacteriaceae. Molecular methods are more accurate for the detection of carbapenemases, but they too require expertise and are expensive. ${ }^{[31]}$

EUCAST recommends that following the routine detection of reduced carbapenem susceptibility, additional phenotypic methods for the detection of carbapenemases should be applied. ${ }^{[14]}$ Combination disc tests (CDTs) are discs or tablets with a carbapenem and various inhibitors. Inhibitors such as boronic acid and dipicolinic acid inhibit class $\mathrm{A}$ and class B carbapenemases, respectively. The AmpC beta-lactamase inhibitor cloxacillin has been included to differentiate between AmpC hyperproduction plus porin loss and carbapenemase production. As class D (OXA-48) inhibitors have not been included in phenotypic panels, resistance to piperacillin/tazobactam and high-level resistance to temocillin (MIC $>128 \mathrm{mg} / \mathrm{mL}$ ) can be used as phenotypic markers. ${ }^{[14]}$ In a 2015 study $^{[16]}$ aiming to develop a diagnostic flowchart using phenotypic screening and confirmation tests for clinical laboratories, the disc diffusion method using meropenem as the antimicrobial agent of choice was favoured for screening, as automated microdilution AST methods lack sensitivity and specificity. The authors support their findings by the fact that disc diffusion is cheap and simple to perform and can be implemented by many laboratories for routine AST. For confirmation of carbapenemases, Maurer et al. ${ }^{[16]}$ explored several options for the various carbapenemases and conclusively decided that a combination of the EUCAST meropenem screening cutoff of $<28 \mathrm{~mm}$ or MIC $>0.125 \mathrm{mg} / \mathrm{mL}^{[14]}$ and ertapenem (or meropenem) aminophenyl boronic acid and EDTA CDTs plus temocillin disc diffusion (or gradient diffusion-based MIC determination) on Mueller-Hinton agar containing cloxacillin showed promise. Considering these published recommendations and the South African Society for Clinical Microbiology's current recommendation of a shift towards implementing the use of EUCAST guidelines in our SA setting, we have adapted the proposed diagnostic flowchart from Maurer et al. ${ }^{[16]}$ and the EUCAST recommendations using EUCAST guidelines ${ }^{[14]}$ for use in routine, academic, reference and national reference laboratories (Fig. 2). This would assist in the identification and detection of CPE for both clinical management and infection prevention and control purposes.

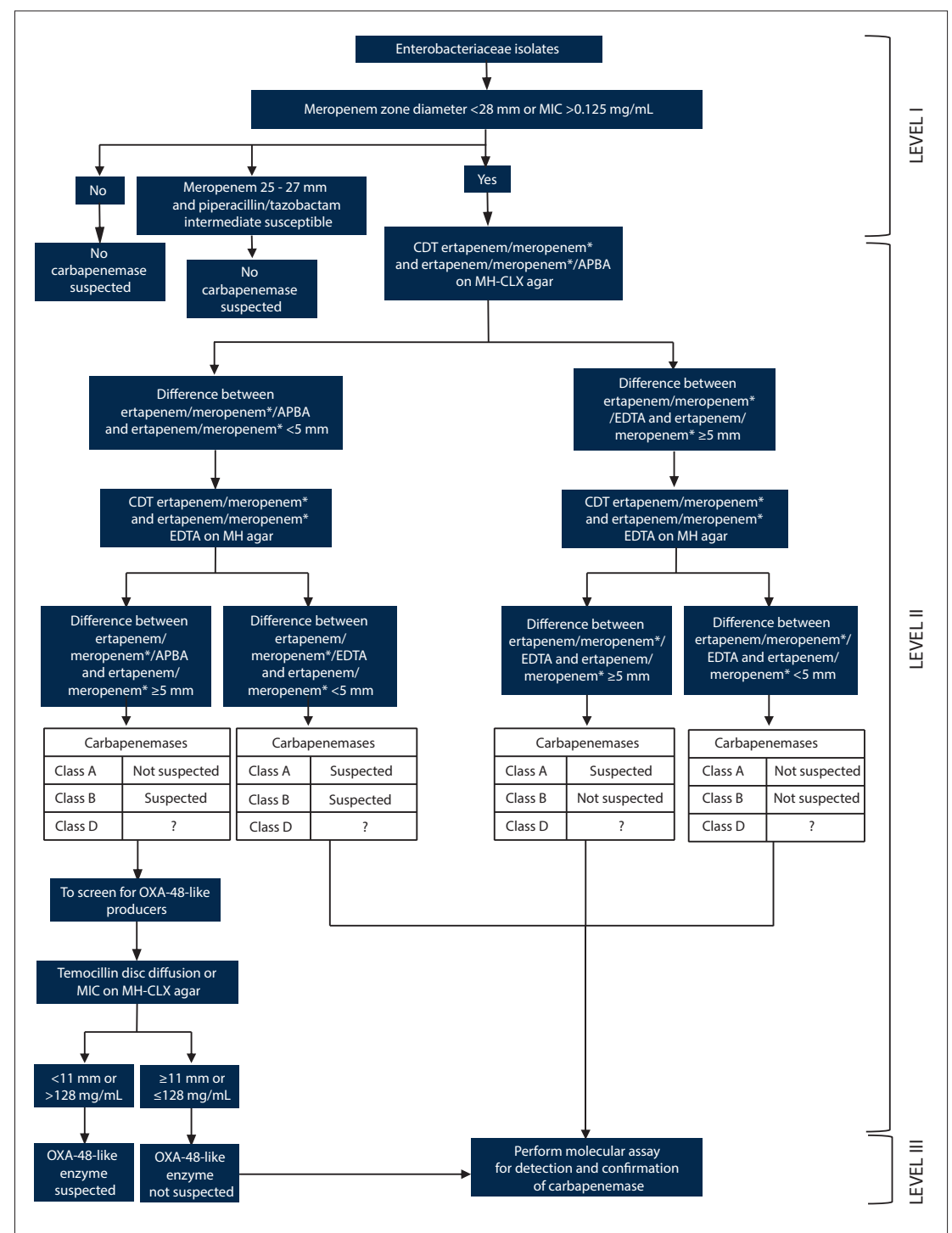

Fig. 2. Diagnostic flow chart for detection of carbapenemases in Enterobacteriaceae, adapted from Maurer et al. ${ }^{[16]}$ and the European Committee on Antimicrobial Susceptibility Testing ${ }^{[14]}$ recommendations. Level I indicates processes to be performed at routine laboratories, level II indicates processes to be performed at academic and reference laboratories, and level III indicates processes to be performed at the national reference laboratory. (MIC = minimal inhibitory concentration; $C D T=$ combined disc test $; A P B A=$ aminophenyl boronic acid; $M H=$ Mueller-Hinton agar; $M H-$ $C L X=$ Mueller-Hinton agar containing cloxacillin; EDTA = ethylenediaminetetra-acetic acid . ${ }^{*}$ Note: meropenem is known to have a lower sensitivity.) 


\section{Conclusions}

We have shown an increase in carbapenemase-producing Enterobacteriaceae over the study period. While phenotypic methods are acceptable for routine diagnosis, clinicians should be aware that carbapenemase-producing isolates are being misidentified as carbapenem-susceptible owing to limitations in routine phenotypic methodology. The application of CLSI and EUCAST clinical breakpoints, although suitable for clinical management, is not ideal for public health and infection prevention and control purposes; the use of epidemiological cut-off values is more appropriate for the latter. However, this is only fitting for use in a region with low CPE prevalence. A comprehensive CPE phenotypic screening method should be implemented to streamline isolates for molecular testing, which is relatively expensive.

Acknowledgements. We thank Ruth Mohlabeng, Wilhelmina Strasheim, Cheryl Hamman, Rubeina Badat, Gloria Molaba, Naseema Bulbulia and Rosah Mabokachaba for assistance with the laboratory work and Boniwe Makwakwa and Penny Crowther-Gibson for assistance with the database. Many thanks to the Centre for Healthcare-associated infections, Antimicrobial Resistance and Mycoses writing group, and particularly Dr Husna Ismail for review of the manuscript.

Author contributions. AS-M conceived and designed the experiments, performed the experiments, analysed the data and wrote the manuscript. OP was instrumental in the study design and its co-ordination and in editing of the manuscript. Both authors read and approved the final manuscript.

Funding. This work was funded by the National Institute for Communicable Diseases. No external funding was obtained.

Conflicts of interest. None.

1. Gupta N, Limbago BM, Patel JB, Kallen AJ. Carbapenem-resistant Enterobacteriaceae: Epidemiology and prevention. Clin Infect Dis 2011:53(1):60-67. https://doi.org/10.1093/cid/cir202

2. Spellberg B, Guidos R, Gilbert D, et al. The epidemic of antibiotic-resistant infections: A call to action for Spellberg B, Guidos R, Gilbert D, et al. The epidemic of antibiotic-resistant infections: A call to action for
the medical community from the Infectious Diseases Society of America. Clin Infect Dis 2008;46(2):155the medical community from the Infections

3. Kocsis B, Szabó D. Antibiotic resistance mechanisms in Enterobacteriaceae. In: Méndez-Vilas A, ed. Microbial Pathogens and Strategies for Combating Them: Science, Technology and Education, Vol. 1. Badajoz, Spain: Formatex Research Center, 2013.

4. Livermore DM. My CRE is a CPO, is yours? Public Health England, Antimicrobial Resistance and Healthcare Associated Infections Reference Unit (AMRHAI) News, Summer 2013. Issue 03:2. PHE publications gateway number: 2013137. http://webarchive.nationalarchives.gov.uk/20140505163041/ http://www.hpa.org.uk/ProductsServices/MicrobiologyPathology/LaboratoriesAndReferenceFacilities/ AntimicrobialResistanceAndHealthcareAssociatedInfections/ARMRLNewsletters/ (accessed 5 May 2018). 5. Livermore DM, Woodford N. The beta-lactamase threat in Enterobacteriaceae, Pseudomonas and Acinetobacter. Trends Microbiol 2006;14(9):413-420. https://doi.org/10.1016/j.tim.2006.07.008

6. Brink A, Coetzee J, Clay C, et al. The spread of carbapenem-resistant Enterobacteriaceae in South Africa: Risk factors for acquisition and prevention. S Afr Med J 2012;102(7):599-601.

7. Sekyere JO, Govinden U, Essak S. The molecular epidemiology and genetic environment of carbapenemases detected in Africa. Microb Drug Resist 2015;22(1):59-68. https://doi.org/10.1089/ mdr.2015.0053

8. Rodriguez-Martinez JM, Cano ME, Velasco C, Martinez-Martinez L, Pascual A. Plasmid-mediated quinolone resistance: An update. J Infect Chemother 2011;17(2):149-182. https://doi.org/10.1007/ s10156-010-0120-2
9. Rodriguez-Martinez JM, Nordmann P, Fortineau N, Poirel L. VIM-19, a metallo-beta-lactamase with increased carbapenemase activity from Escherichia coli and Klebsiella pneumoniae. Antimicrob Agents increased carbapenemase activity from Escherichia coli and Klebsiella p
Chemother 2010;54(1):471-476. https://doi.org/10.1128/AAC.00458-09

10. Fernández-Cuenca F, Rodríguez-Martínez IM, Gómez-Sánchez MA, de Alba PD, Infante-Martínez

V, Pascual A. Production of a plasmid-encoded OXA-72 $\beta$-lactamase associated with resistance to
Vintinez carbapenems in a clinical isolate Acinetobacter junii. Int J Antimicrob Agents 2012;39(1):93-94. https:// doi.org/10.1016/.ijantimicag.2011.07.017

11. Chea N, Bulens SN, Kongphet-Tran T, et al. Improved phenotype-based definition for identifying carbapenemase producers among carbapenem-resistant Enterobacteriaceae. Emerg Infect Dis 2015;21(9):1611-1616. https://doi.org/10.3201/eid2109.150198

12. Hayakawa K, Miyoshi-Akiyama T, Kirikae T, et al. Molecular and epidemiological characterization of IMP-type metallo-beta-lactamase-producing Enterobacter cloacae in a large tertiary care hospital in Japan. Antimicrob Agents Chemother 2014;58(6):3441-3450. https://doi.org/10.1128/AAC.02652-13

13. Singh-Moodley A, Ekermans P, Perovic O. Emerging carbapenem-pesistant Enterobacter cloacae producing OXA-48-, VIM- and IMP-type- $\beta$-lactamases in Eastern Cape hospitals in South Africa. Open J Med Microbiol 2015;5(4):246-253. https://doi.org/10.4236/ojmm.2015.54030

14. European Committee on Antimicrobial Susceptibility Testing (EUCAST). EUCAST guidelines for dean guidelines for detection of restance
importance. 2017. http://www.eucast.org/fileadmin/src/media/PDFs/EUCAST files/Resistance mechanisms/EUCAST_detection_of_resistance_mechanisms_170711.pdf (accessed 5 May 2018).

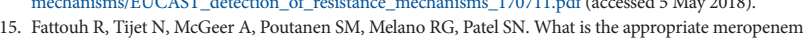
. Fattouh R, Tijet N, McGeer A, Poutanen SM, Melano RG, Patel SN. What is the appropriate meropenen
MIC for screening of carbapenemase-producing Enterobacteriaceae in low-prevalence settings? MIC for screening of carbapenemase-producing Enterobacteriaceae in low-prevalence
Antimicrob Agents Chemother 2016;60(3):1556-1559. https://doi.org/10.1128/AAC.02304-15

16. Maurer FP, Castelberg C, Quiblier C, Bloemberg GV, Hombach M. Evaluation of carbapenemase screening and confirmation tests with Enterobacteriaceae and development of a practical diagnostic algorithm. J Clin Microbiol 2015;53(1):95-104. https://doi.org/10.1128/JCM.01692-14

17. Nordmann P, Gniadkowski M, Giske CG, et al. Identification and screening of carbapenemase-producing Enterobacteriaceae. Clin Microbiol Infect 2012;18(5):432-438.

18. Richter SS, Marchaim D. Screening for carbapenem-resistant Enterobacteriaceae: Who, when and how? Virulence 2017;8(4):417-426. https://doi.org/10.1080/21505594.2016.1255381

19. Clinical and Laboratory Standards Institute. Performance standards for antimicrobial susceptibility testing: Twenty seventh informational supplement. 2017. Wayne, Penn., USA: CLSI, 2017.

20. European Committee on Antimicrobial Susceptibility Testing (EUCAST). Breakpoint tables for European Committee on Antimicrobial Susceptibility Testing (EUCAST). Breakpoint tables for
interpretation of MICs and zone diameters. Version 7.1, 2017. http://www.eucast.org/fileadmin/src/ media/PDFs/EUCAST_files/Breakpoint_tables/v_7.1_Breakpoint_Tables.pdf(accessed 5 May 2018).

21. Brink AJ, Coetzee J, Clay CG, et al. Emergence of New Delhi metallo-beta-lactamase (NDM-1) and Krink Af, Coetzee J, Clay CG, et al. Emergence of New Delhi metallo-beta-lactamase (NDM-1) and
Klebseumoniae carbapenemase (KPC-2) in South Africa. J Clin Microbiol 2011;50(2):525-527. Klebsiella pneumoniae carbapenemase
https://doi.org/10.1128/JCM.05956-11

22. Brink AJ, Coetzee J, Corcoran C, et al. Emergence of OXA-48 and OXA-181 carbapenemases among Enterobacteriaceae in South Africa and evidence of in vivo selection of colistin resistance as a consequence of selective decontamination of the gastrointestinal tract. J Clin Microbiol 2013;51(1):369372. https://doi.org/10.1128/JCM.02234-12

23. Govind C, Moodley K, Peer AK, et al. NDM-1 imported from India - first reported case in South Africa. S Afr Med J 2013;103(7):476-478. https://doi.org/10.7196/SAMJ.6593

24. Jacobson RK, Manesen MR, Moodley C, et al. Molecular characterisation and epidemiological investigation of an outbreak of $b l a_{\text {OXA-181 }}$ carbapenemase-producing isolates of Klebsiella pneumoniae in South Africa. S Afr Med J 2015;105(12):1030-1035. https://doi.org/10.7196/SAMJ.2015.v105i12.9926

25. Lowman W, Sriruttan C, Nana T, et al. NDM-1 has arrived: First report of carbapenem resistance mechanism in South Africa. S Afr Med J 2011;101(12):873-875.

26. Mimoz O, Gregoire N, Poirel L, Marliat M, Couet W, Nordmann P. Broad-spectrum beta-lactam antibiotics for treating experimental peritonitis in mice due to Klebsiella pneumoniae producing the antibiotics for treating experimental peritonitis in mice due to Klebsiella pneumoniae producing the
carbapenemase OXA-48. Antimicrob Agents Chemother 2012;56(5):2759-2760. https://doi.org/10.1128 carbapenemase

AAC.06069-11
27. Glupczynski Y, Huang TD, Bouchahrouf W, et al. Rapid emergence and spread of OXA-48-producing carbapenem-resistant Enterobacteriaceae isolates in Belgian hospitals. Int I Antimicrob Agent 2012;39(2):168-172. https://doi.org/10.1016/j.ijantimicag.2011.10.005

28. Girlich D, Poirel L, Nordmann P. Value of the modified Hodge test for detection of emerging carbapenemases in Enterobacteriaceae. J Clin Microbiol 2012;50(2):477-479. https://doi.org/10.1128/ JCM.05247-11

29. Singh-Moodley A, Perovic O. Antimicrobial susceptibility testing in predicting the presence of carbapenemase genes in Enterobacteriaceae in South Africa. BMC Infect Dis 2016;16(536). https://doi. org/10.1186/s12879-016-1858-7

30. European Committee on Antimicrobial Susceptibility Testing (EUCAST). EUCAST guidelines for detection of resistance mechanisms and specific resistances of clinical and/or epidemiological importance. 2013. http:// www.eucast.org/fileadmin/src/media/PDFs/EUCAST_files/Resistance_mechanisms/EUCAST_detection of_resistance_mechanisms_v1.0_20131211.pdf (accessed: 5 May 2018).

of_resistance_mechanisms_v1.0_20131211.pdf (accessed: 5 May 2018).
31. Lutgring JD, Limbago BM. The problem of carbapenemase-producing-carbapenem-resistant Enterobacteriaceae detection. J Clin Microbiol 2016;54(3):529-534. https://doi.org/10.1128/JCM.02771-15

Accepted 6 November 2017. 